\title{
Parámetros ecográficos de los órganos abdominales del tamandúa (Tamandua tetradactyla)
}

\author{
Ultrasound parameters of the abdominal organs of tamandua \\ (Tamandua tetradactyla)
}

\author{
Jenny Dupont T. ${ }^{1}$, Ricardo Grandez R. ${ }^{1,2}$, Catalina Hermoza G. ${ }^{1}$
}

\section{Resumen}

\begin{abstract}
El tamandúa (Tamandua tetradactyla) es un mamífero que pertenece al superorden Xenarthra, familia Myrmecophagidae, animales solitarios y de hábitos crepusculares. El objetivo del estudio fue describir la topografía, morfología y ubicación de los órganos abdominales mediante una evaluación ecográfica. Se emplearon cinco especímenes de $T$. tetradactyla criados en cautiverio del Parque Zoológico Huachipa, Lima, Perú (tres machos adultos y dos juveniles), en aparente buen estado de salud. Se utilizó un ecógrafo Pie Medical modelo 100 Falco Vet ${ }^{\circledR}$ con transductor convexo multifrecuencial de 5-7.5 $\mathrm{MHz}$. Se evaluó la topografía, márgenes, forma, dimensiones, ecotextura y ecogenicidad de los órganos abdominales. Los resultados obtenidos no difieren de los encontrados en individuos de especie canina de la misma talla y peso. Sin embargo, se ha encontrado que el tamandúa, al igual que el oso perezoso (Choloepus hoffmanni), tiene los testículos intraabdominales y los riñones ubicados entre las regiones del flanco e inguinal.
\end{abstract}

Palabras clave: ecografía; Xenarthra; Tamandua tetradactyla; órganos abdominales

\section{Abstract}

Tamandua (Tamandua tetradactyla) is a mammal that belongs to the superorder Xenarthra, family Myrmecophagidae, lonely animal with crepuscular habits. The objective of the study was to describe the topography, morphology, and location of the abdominal organs through an ultrasound evaluation. Five specimens of T. tetradactyla in captivity from the Huachipa Zoological Park, Lima, Peru (three adult males and two juveniles), in

1 Sección de Biociencias y Ciencias Clínicas, Departamento Académico de Medicina Veterinaria y Zootecnia, Facultad de Medicina Veterinaria y Zootecnia, Universidad Peruana Cayetano Heredia, Lima, Perú

2E-mail: rgrandez@hotmail.com

Recibido: 27 de noviembre de 2017

Aceptado para publicación: 16 de abril de 2018 
apparent good health conditions were used. A Pie Medical Ultrasound Falco Vet ${ }^{\circledR}$ Model 100 with a multifrequency convex transducer of 5-7.5 MHz was used. The topography, margins, shape, dimensions, echotexture and echogenicity of the abdominal organs were evaluated. The results did not differ from those found in dogs of the same size and weight. However, it has been found that the tamandua, like the Hoffmann's two-toed sloth lazy bear (Choloepus hoffmanni), has intra-abdominal testicles and the kidneys located between the flank and inguinal regions.

Key words: ultrasound; Xenarthra; Tamandua tetradactyla; abdominal organs

\section{INTRODUCCIÓN}

El tamandúa es un mamífero que forma parte del Superorden Xenarthra (anteriormente llamado Edentata) que se agrupa en dos órdenes: Cingulata (armadillos) y Pilosa (osos hormigueros y perezosos). Los tamandúas pertenecen al Orden Pilosa, Suborden Vermilingua y Familia Myrmecophagidae (Gardner, 2007). Edentata o Edentado significa que carece de dientes, y solo la familia Mymercophagidae (osos hormigueros, incluyendo tamandúas) son propiamente desdentados.

Existen dos especies de tamandúa, el Tamandua mexicana, que se encuentra desde el sureste de México hasta el suroeste de América del Sur, y el Tamandua tetradactyla, que está presente desde el noroeste de Venezuela hasta el norte de Argentina y Uruguay, incluyendo la isla de Trinidad, las Guayanas, Brasil, Uruguay y Paraguay, así como la cuenca occidental del Amazonas de Colombia, Ecuador, Perú y Bolivia (Gardner, 2007).

El tamandúa (Tamandua tetradactyla) se denomina en general como oso hormiguero, pero además recibe el nombre de oso melero y oso hormiguero de collar (Superina et al., 2010). En general, los llamados osos hormigueros se caracterizan morfológicamente por presentar un cráneo alargado y estrecho, una lengua larga cubierta con papilas, con saliva pegajosa y patas delante- ras de gran alcance con garras largas y curvas (Alves da Rosa et al., 2010). Poseen pobre visión y un excelente olfato. Son considerados como animales nocturnos o crepusculares solitarios (Gardner, 2007), predominantemente arbóreos, pero también se mueven, alimentan y descansan en el suelo (Superina et al., 2008). Son altamente especializados en su nutrición, alimentándose casi exclusivamente de termitas (Nasutitermes spp) y hormigas (Crematogaster spp y Camponotus spp), siendo el resto de la dieta abejas, heterópteras, pupas de insectos y semillas (Valdes y Brenes, 2012; Pérez y Gonzales, 2004). La temperatura corporal de los edentados es más baja que la de los demás mamíferos (Vogt y Becker, 1987; Fernandes y Young, 2008), debido a una baja tasa metabólica (Valdes y Brenes, 2012).

Los tamandúas tienen un intestino delgado largo, 7.8 veces mayor que el intestino grueso, con un tamaño medio aproximado de $3.5 \mathrm{~m}$; el duodeno es de corta longitud con un tamaño medio de $15 \mathrm{~cm}$; el yeyuno y el íleon constituyen la mayor parte del intestino con cerca de $3.4 \mathrm{~m}$, representando al $96 \%$ del intestino delgado. Todo el intestino posee un tamaño apro-ximado de $4.0 \mathrm{~m}$ (Macedo et al., 2011).

El útero es simple y alargado, de forma piriforme y situado en la entrada de la cavidad pélvica. Los cuernos uterinos se encuentran encorvados hacia los ovarios, siendo alargados y posicionados paralelos al útero (Bonatelli et al., 2003). El útero de un animal 
gestante mide entre 8 a $13 \mathrm{~cm}$ y presenta una placa discoidal blanquecina. La placenta es hemocorial discoidal y lobulada. La zona anogenital, en los dos sexos, es similar. El clítoris de una hembra joven está cubierto por un par de labios vulvares gruesos cubierto de pelos (Hayssen, 2011). Los principales problemas de salud en osos hormigueros en cautiverio, tanto en las especies tamandúa como en el oso hormiguero gigante (Myrmecophaga tridactyla) son los trastornos del sistema digestivo, deficiencias nutricionales y presencia de heridas (Diniz et al., 1995).

La imagenología es un medio no invasivo de diagnóstico para ver estructuras internas y su función en vivo, pudiendo identificarse tejidos anormales y patologías como las neoplasias (Zanzonico, 2011); además, permiten diferenciar entre tipos de tejido blando y la arquitectura interna con detalle del órgano (Barr, 2006). La ultrasonografía tiene una variedad de usos en animales exóticos, como son determinar el sexo y el estado reproductivo del animal, evaluar estructuras y órganos anormales, y realizar biopsias guiadas para órganos o lesiones específicas (Mannion, 2006).

Existen antecedentes de una investigación sobre diagnóstico ultrasonográfico de gestación en $T$. tetradactyla (Soto et al., 2006); sin embargo, no existen estudios publicados sobre ultrasonografía abdominal en esta especie. El objetivo del presente estudio fue contar con información de los parámetros y características ecográficas normales de los órganos abdominales, información que permitirá identificar alteraciones en estos órganos e implementar procedimientos terapéuti$\cos$, quirúrgicos y profilácticos.

\section{Materiales y Métodos}

El tamaño de muestra correspondió al total de ejemplares de la especie $T$. tetradactyla criados en cautiverio en el Parque Zoológico Huachipa (distrito de Ate
Vitarte - Lima, Perú). La población consistía en tres machos adultos y dos juveniles (hembra y macho). Se confirmó que los animales tenían buen estado de salud al examen físico completo (realizado previo a la sedación) y que no tenían reportes de tratamientos o enfermedades previas en los últimos tres meses. Durante la sedación se tomó una muestra de sangre de la vena coccígea para realizar el hemograma completo para verificar su estado de salud.

Los animales fueron sometidos a ayuno de 24 horas previas al estudio ecográfico. Se pesaron e identificaron para luego proceder a aplicar los fármacos anestésicos vía intramuscular. La contención y anestesia de los animales se realizó de acuerdo con el protocolo convencional para $T$. tetradactyla del Parque Zoológico Huachipa, adaptado de Moreno (2012), el cual incluía ketamina $(4.0 \pm 0.25 \mathrm{mg} / \mathrm{kg})$, midazolam $(0.1 \mathrm{mg} / \mathrm{kg})$ y dexmedetomidina $(20 \pm 5 \mathrm{mg} / \mathrm{kg})$

El animal se posicionó decúbito dorsal para el examen ecográfico y se aplicó gel en la zona abdominal para un correcto contacto con el transductor. Se utilizó un equipo de Ultrasonido Pie Medical modelo 100 Falco Vet ${ }^{\circledR}$ con transductor convexo multifrecuencial de 5-7.5 MHz. Las imágenes fueron documentadas mediante la impresora Video Graphic Printer Sony UP-895 MD® utilizando papel térmico Type II - Upp 110 D.

Para la mejor descripción de la topografía se empleó la metodología de estudio descrita por Howard y De Lahunta (1991), que consiste en una división imaginaria de la superficie abdominal en tres zonas: abdomen craneal (epigastrio), abdomen medio (mesogastrio) y abdomen caudal (hipogastrio) y en nueve regiones: xifoidea, hipocondrio derecho, hipocondrio izquierdo, umbilical, flanco derecho, flanco izquierdo, púbica, inguinal derecha e inguinal izquierda (ver Figura 1).

El examen ecográfico se inició visualizando el hígado y vesícula biliar, en la parte craneal del abdomen; continuando con- 
la evaluación del bazo y estómago, en el abdomen medio. Se prosiguió con la evaluación del riñón izquierdo y derecho, y la vejiga urinaria en la zona caudal de la cavidad abdominal. Luego se evaluaron los órganos reproductivos, ubicándolos en la parte profunda de la pelvis. En todo momento se tomó nota de la topografía, morfología, ecogenicidad, ecotextura y dimensiones, así como otros datos particulares hallados durante el examen de cada órgano.

Los resultados de las dimensiones de los órganos registrados fueron evaluados utilizando la media como medida de tendencia central y la desviación estándar y rango como medidas de dispersión. La topografía, morfología, ecogenicidad y ecotextura fueron descritas individualmente y ordenadas en cuadros para facilitar su interpretación.

\section{Resultados}

Al examen físico de los animales, realizado por el médico veterinario del parque zoológico, no se encontraron alteraciones específicas. Los resultados de las constantes vitales durante el examen físico y de los parámetros del hemograma estuvieron dentro del rango normal descrito para la especie (Sanches et al., 2013), con excepción de la hembra juvenil que mostró una anemia regenerativa leve y leucocitosis leve. Asimismo, cabe notar que no se pudo colectar suficiente cantidad de muestra en el espécimen juvenil macho para realizar el hemograma.

La evaluación ultrasonográfica de los especímenes adultos no presentó ninguna dificultad, pudiéndose obtener las dimensiones

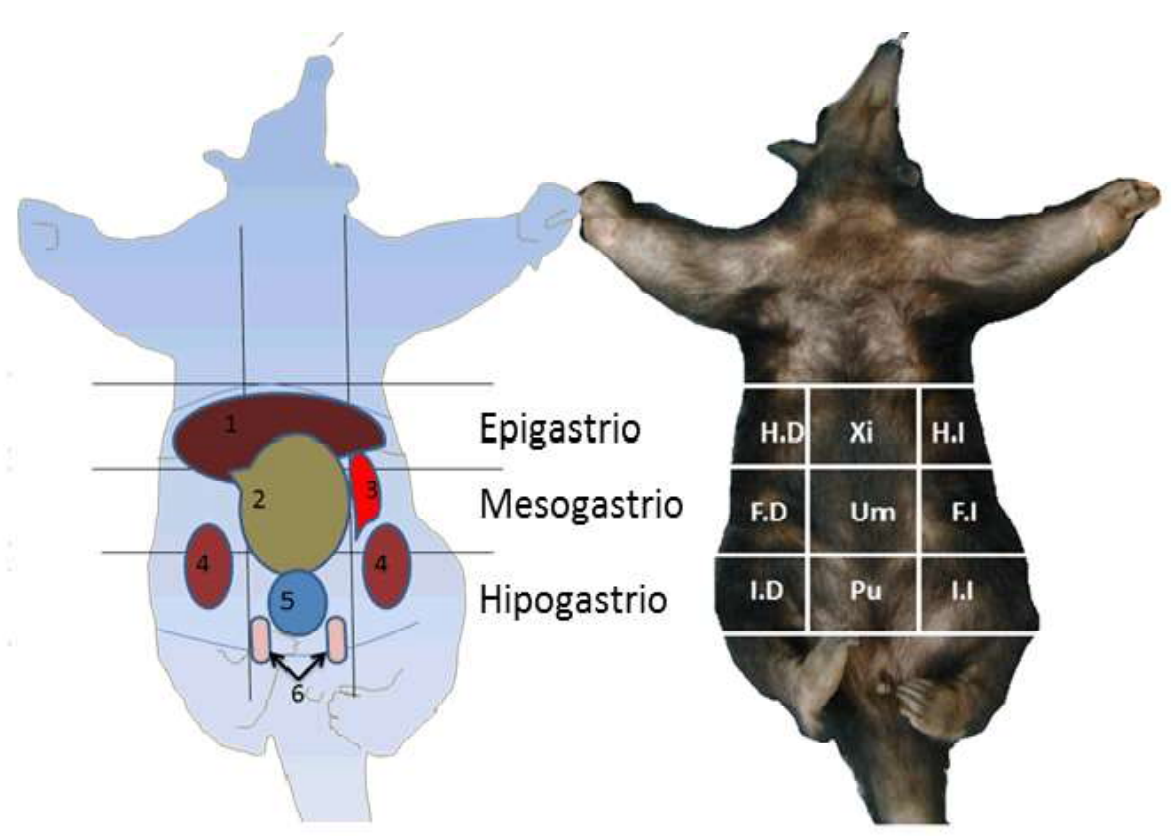

Figura 1. Esquema de las zonas, regiones y topografía de los órganos abdominales del tamandúa (Tamandua tetradactyla). Se puede apreciar las siguientes zonas y regiones: $\mathrm{Xi}$, xifoidea; $\mathrm{HD}$, hipocondrio derecho; $\mathrm{HI}$, hipocondrio izquierdo: Um, umbilical; FD, flanco derecho; Pu, púbica; ID, inguinal derecha; II, inguinal izquierdo; y la ubicación topográfica de los órganos: 1, hígado; 2, estomago; 3, bazo; 4 , riñones; 5 , vejiga; 6 , testículos 
Cuadro 1. Dimensiones promedio y desvío estándar (D.E.) mediante ecografía de órganos abdominales de cinco ejemplares de Tamandua tetradactyla del Parque Zoológico Huachipa (Lima, Perú) (Parte 1)

\begin{tabular}{|c|c|c|c|c|c|c|c|c|c|}
\hline \multirow{3}{*}{ Espécimen } & \multirow{3}{*}{ Sexo } & \multirow{3}{*}{$\begin{array}{c}\text { Edad } \\
\text { (años) }\end{array}$} & \multirow{3}{*}{$\begin{array}{c}\text { Vesícula } \\
\text { (largo, } \\
\text { cm) }\end{array}$} & \multirow{3}{*}{$\begin{array}{c}\text { Estómago } \\
\text { (grosor } \\
\text { pared, } \\
\mathrm{cm} \text { ) }\end{array}$} & \multirow{3}{*}{$\begin{array}{c}\text { Bazo } \\
\text { (espeso, } \\
\mathrm{cm})\end{array}$} & \multicolumn{4}{|c|}{ Riñones (cm) } \\
\hline & & & & & & \multicolumn{2}{|c|}{ Izquierdo } & \multicolumn{2}{|c|}{ Derecho } \\
\hline & & & & & & Largo & Ancho & Largo & Ancho \\
\hline Adulto 01 & $\mathrm{M}$ & 2.0 & 1.51 & 0.27 & 1.35 & 4.57 & 1.68 & 4.35 & 1.55 \\
\hline Adulto 02 & M & 6.0 & 1.42 & 0.20 & 0.98 & 4.14 & 2.48 & 4.12 & 2.47 \\
\hline Adulto 03 & M & 3.0 & 2.27 & 0.20 & 0.99 & 3.78 & 2.26 & 3.98 & 2.30 \\
\hline Media & & & & 0.20 & 1.10 & 4.20 & 2.10 & 4.20 & 2.10 \\
\hline (D.E.) & & & 0.47 & 0.04 & 0.21 & 0.40 & 0.41 & 0.19 & 0.49 \\
\hline Juvenil 01 & M & 0.5 & 0.88 & 0.10 & 0.80 & 3.18 & 1.50 & 2.83 & 1.56 \\
\hline Juvenil 02 & $\mathrm{H}$ & 0.5 & 1.00 & 0.10 & 0.80 & 2.48 & 1.10 & 2.49 & 0.89 \\
\hline Media & & & 0.94 & 0.10 & 0.80 & 2.83 & 1.30 & 2.66 & 1.22 \\
\hline (D.E.) & & & 0.10 & 0.00 & 0.00 & 0.50 & 0.30 & 0.20 & 0.50 \\
\hline
\end{tabular}

de los órganos abdominales: hígado, bazo, estómago, riñones, testículos y vejiga (Cuadros 1 y 2). Los valores de frecuencia (5.0 $7.5 \mathrm{MHz})$ del transductor del equipo ecográfico y el pequeño tamaño de los animales juveniles no permitió establecer las dimensiones de testículos en el macho juvenil y del útero en la hembra juvenil. Las características ultrasonográficas de los órganos se pueden apreciar en el Cuadro 3. La ubicación topográfica de los órganos evaluados se muestra en la Figura 1 y las imágenes ultrasonográficas en la Figura 2. No se encontró alteraciones compatibles con patologías en los órganos durante la evaluación ecográfica.

\section{Discusión}

La posición decúbito dorsal facilitó la evaluación ultrasonográfica de los órganos del tamandúa debido a la forma corporal del animal, que es aplanada dorsoventralmente, requiriéndose únicamente abundante aplicación de gel ultrasonográfico. Los animales se encontraban en buen estado de salud, aspecto que fue corroborado 45 días posteriores al procedimiento ecográfico.

El espécimen juvenil hembra que presentó anemia leve regenerativa no mostró ninguna alteración evidente en el examen físico previo y posterior al procedimiento. Por otro lado, es importante mencionar que los valores hematológicos de la especie no se encuentran bien definidos y la anemia de especímenes en cautiverio puede ser atribuible a estrés o problemas nutricionales (Miranda et al., 2008).

Ante la ausencia de información ecográfica en esta especie no fue posible comparar los resultados obtenidos con animales del mismo género; sin embargo, se consideró comparar las dimensiones y ubicación de los órganos con individuos del mismo superorden Xenarthra, tales como el oso perezoso de dos dedos (Choloepus hoffmanni) 
Cuadro 2. Dimensiones promedio y desvío estándar (D.E.) mediante ecografía de órganos abdominales de cinco ejemplares de Tamandua tetradactyla del Parque Zoológico Huachipa (Lima, Perú) (Parte 2)

\begin{tabular}{|c|c|c|c|c|c|c|c|}
\hline \multirow{3}{*}{ Espécimen } & \multirow{3}{*}{ Sexo } & \multirow{3}{*}{$\begin{array}{c}\text { Edad } \\
\text { (años) }\end{array}$} & \multirow{3}{*}{$\begin{array}{c}\text { Vejiga } \\
\text { (grosor } \\
\text { pared, cm) }\end{array}$} & \multicolumn{4}{|c|}{ Testículos $(\mathrm{cm})$} \\
\hline & & & & \multicolumn{2}{|c|}{ Izquierdo } & \multicolumn{2}{|c|}{ Derecho } \\
\hline & & & & Largo & Ancho & Largo & Ancho \\
\hline Adulto 01 & $\mathrm{M}$ & 2.0 & 0.15 & 2.12 & 1.32 & 2.14 & 1.34 \\
\hline Adulto 02 & M & 6.0 & 0.15 & 3.85 & 1.54 & 3.85 & 1.55 \\
\hline Adulto 03 & M & 3.0 & 0.15 & 3.58 & 1.79 & 3.30 & 1.70 \\
\hline Media & & & 0.15 & 3.20 & 1.60 & 3.10 & 1.50 \\
\hline D.S. & & & 0.00 & 0.93 & 0.24 & 0.87 & 0.18 \\
\hline Juvenil 01 & M & 0.5 & 0.1 & & & & \\
\hline Juvenil 02 & $\mathrm{H}$ & 0.5 & 0.1 & & & & \\
\hline Media & & & 0.1 & & & & \\
\hline D.S & & & 0.0 & & & & \\
\hline
\end{tabular}
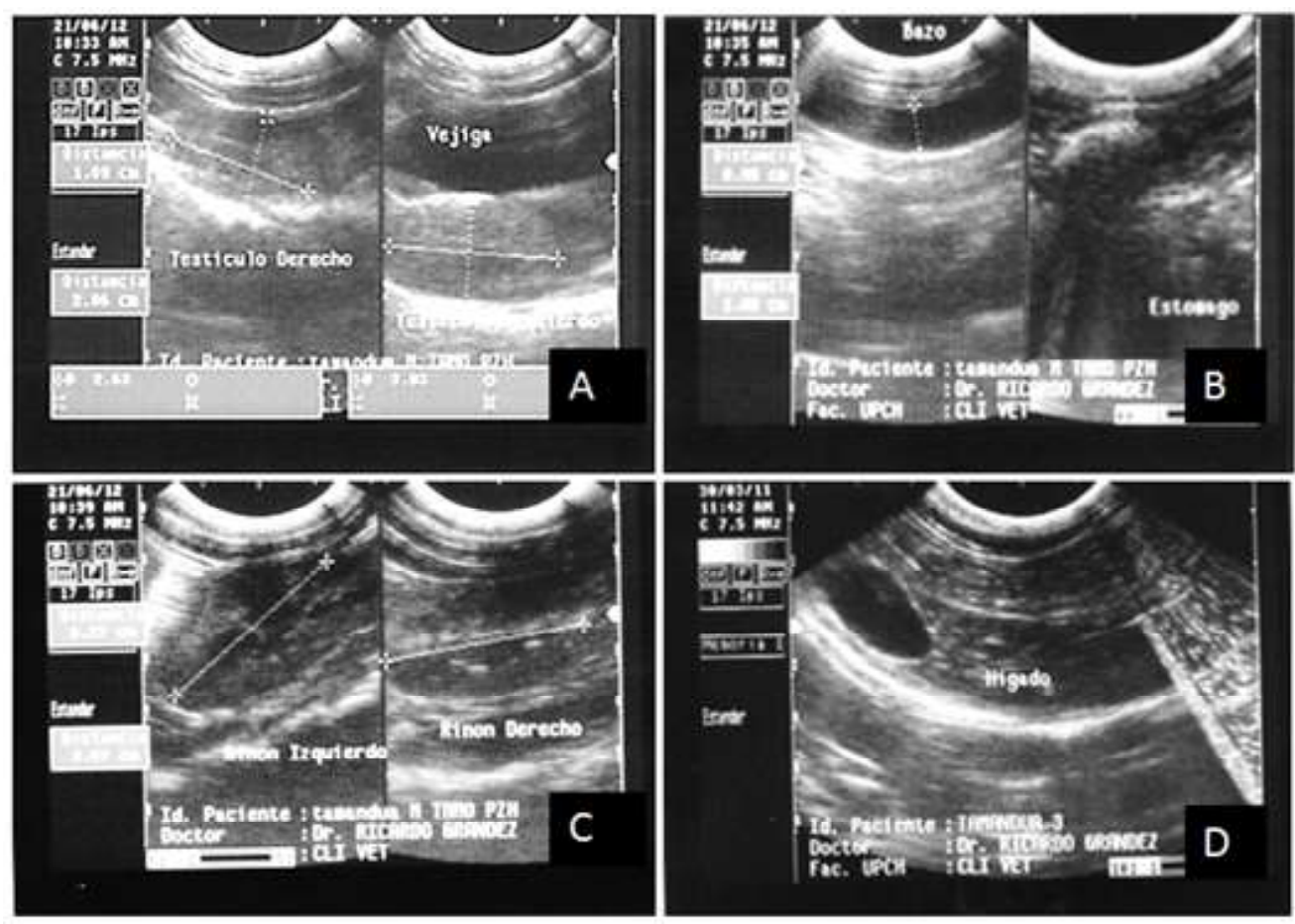

Figura 2. Imágenes ecográficas de tamandúas (Tamandua tetradactyla) del Parque Zoológico Huachipa (Lima, Perú), mostrando dimensiones de órganos abdominales. A: testículos derecho e izquierdo y vejiga. B: bazo y estómago. C: riñones izquierdo y derecho. D: hígado y vesícula biliar 
Cuadro 3. Características ecográficas: topografía, márgenes, forma, ecotextura y ecogenicidad de los órganos abdominales evaluados en cinco ejemplares de Tamandua tetradactyla. Parque Zoológico Huachipa (Lima, Perú)

\begin{tabular}{|c|c|c|c|c|c|c|}
\hline & Bazo & Estómago & Riñón & Hígado & Vejiga & Testículos \\
\hline Número & Único & Único & Doble & Único & Único & Doble \\
\hline $\begin{array}{l}\text { Topografía } \\
\text { (región) }\end{array}$ & $\begin{array}{l}\text { Flanco } \\
\text { izquierdo }\end{array}$ & $\begin{array}{l}\text { Xifoides y } \\
\text { umbilical } \\
\text { craneal }\end{array}$ & $\begin{array}{l}\text { Entre flanco } \\
\text { e inguinal } \\
\text { correspon- } \\
\text { diente }\end{array}$ & $\begin{array}{l}\text { Xifoidea e } \\
\text { hipocondrio } \\
\text { derecho e } \\
\text { izquierdo. } \\
\text { Vesícula biliar } \\
\text { en parénquima } \\
\text { hepático } \\
\text { hipocondrio } \\
\text { derecho }\end{array}$ & $\begin{array}{l}\text { Región } \\
\text { púbica }\end{array}$ & $\begin{array}{l}\text { Intra- } \\
\text { abdominal, } \\
\text { límite de } \\
\text { región } \\
\text { púbica e } \\
\text { inguinal } \\
\text { correspon- } \\
\text { diente }\end{array}$ \\
\hline Márgenes & $\begin{array}{l}\text { Bordes } \\
\text { continuos } \\
\text { redondos, } \\
\text { cápsula } \\
\text { ecogénica } \\
\text { definida }\end{array}$ & $\begin{array}{l}\text { Márgenes } \\
\text { externos } \\
\text { regulares, } \\
\text { interno } \\
\text { ondulante }\end{array}$ & $\begin{array}{l}\text { Bordes } \\
\text { definidos, } \\
\text { cápsula } \\
\text { ecogénica } \\
\text { regular }\end{array}$ & $\begin{array}{l}\text { Regulares y } \\
\text { continuos. } \\
\text { Vesícula de } \\
\text { bordes delgados, } \\
\text { eventualmente } \\
\text { discontinuos }\end{array}$ & $\begin{array}{l}\text { Bordes } \\
\text { gruesos } \\
\text { regulares y } \\
\text { ecogénicos }\end{array}$ & $\begin{array}{l}\text { Bordes } \\
\text { regulares, } \\
\text { cápsula } \\
\text { ecogénica }\end{array}$ \\
\hline Forma & $\begin{array}{l}\text { Alargado o } \\
\text { de forma } \\
\text { triangular } \\
\text { según la } \\
\text { distención } \\
\text { gástrica }\end{array}$ & $\begin{array}{l}\text { Sacular e } \\
\text { irregular } \\
\text { dependiendo } \\
\text { del estado de } \\
\text { distención. }\end{array}$ & $\begin{array}{l}\text { Ovalados. } \\
\text { Relación } \\
\text { corteza } \\
\text { medula } 1: 1 .\end{array}$ & $\begin{array}{l}\text { Varios lóbulos } \\
\text { que se unen en } \\
\text { una sola } \\
\text { estructura } \\
\text { ovalada con } \\
\text { predominio } \\
\text { derecho. }\end{array}$ & Sacular & $\begin{array}{l}\text { Ovalados, y } \\
\text { alargados } \\
\text { cráneo- } \\
\text { caudalmente }\end{array}$ \\
\hline Ecotextura & $\begin{array}{l}\text { Homogéneo } \\
\text { y granulari- } \\
\text { dad fina a } \\
\text { mediana }\end{array}$ & $\begin{array}{l}\text { Mucosa de } \\
\text { disposición } \\
\text { en capas, con } \\
\text { capa ondeada } \\
\text { (dependiendo } \\
\text { de estado de } \\
\text { distención) }\end{array}$ & $\begin{array}{l}\text { Corteza } \\
\text { homogénea } \\
\text { de } \\
\text { granularidad } \\
\text { media y } \\
\text { médula con } \\
\text { espacios } \\
\text { irregulares } \\
\text { anecoicos }\end{array}$ & $\begin{array}{l}\text { Homogéneo de } \\
\text { granularidad } \\
\text { media con } \\
\text { presencia de } \\
\text { estructuras } \\
\text { lineales cortas } \\
\text { (vasos } \\
\text { sanguíneos) }\end{array}$ & $\begin{array}{l}\text { Mucosa de } \\
\text { disposición } \\
\text { en capas, } \\
\text { con interna } \\
\text { levemente } \\
\text { irregular } \\
\text { según la } \\
\text { distención }\end{array}$ & $\begin{array}{l}\text { Granular } \\
\text { mediana } \\
\text { homogénea }\end{array}$ \\
\hline $\begin{array}{l}\text { Ecogenici- } \\
\text { dad }\end{array}$ & $\begin{array}{l}\text { Hipereco- } \\
\text { génico en } \\
\text { relación con } \\
\text { corteza } \\
\text { renal } \\
\text { izquierda }\end{array}$ & $\begin{array}{l}\text { Alternancia } \\
\text { de ecogeni- } \\
\text { cidad entre } \\
\text { capas de la } \\
\text { mucosa }\end{array}$ & $\begin{array}{l}\text { La corteza } \\
\text { hipoeco- } \\
\text { génica } \\
\text { respecto a } \\
\text { bazo e } \\
\text { hígado. } \\
\text { Médula } \\
\text { presenta } \\
\text { predominio } \\
\text { anecoico }\end{array}$ & $\begin{array}{l}\text { Contenido de } \\
\text { vesícula } \\
\text { anecoico. } \\
\text { Parénquima } \\
\text { hepático } \\
\text { hipoecogénico } \\
\text { con relación a } \\
\text { bazo; y similar o } \\
\text { levemente } \\
\text { hiperecogénico } \\
\text { con relación a } \\
\text { corteza renal }\end{array}$ & $\begin{array}{l}\text { Alternancia } \\
\text { ecogénica } \\
\text { entre capas } \\
\text { de mucosa } \\
\text { con } \\
\text { contenido } \\
\text { anecoico }\end{array}$ & $\begin{array}{l}\text { Levemente } \\
\text { hipereco- } \\
\text { génico en } \\
\text { relación al } \\
\text { bazo }\end{array}$ \\
\hline
\end{tabular}


y el perro doméstico (Canis lupus familiaris), al poseer algunas características físicas y anatómicas parecidas o ser una especie mejor estudiada. Ante esto, las dimensiones de los órganos abdominales de $T$. tetradactyla, considerando que son animales adultos con pesos entre 7 y $10 \mathrm{~kg}$ y juveniles entre 1 y $2 \mathrm{~kg}$, no difirieron de lo observado en los caninos de similar tamaño y peso (Gomes, 2004; Halásc, 2004; Mamprim, 2004; Janthur y Lüerssen, 2010).

La medida del largo y ancho de los riñones y la relación entre estas dos medidas en la población estudiada es similar a lo que descrito por Janthur y Lüerssen (2010) para esas medidas en caninos. El promedio del largo de los riñones para perros de 5-9 kg (Barr, 2006) es semejante al largo de los riñones de tamandúas. La forma ovalada del riñón guarda similitud con la de C. hoffmanni (Salgado, 2010) y de los caninos (Janthur y Lüerssen, 2010); en tanto que, la ubicación de los riñones difiere de la topografía de los caninos, ubicándose entre la región del flanco e inguinal; ubicación que comparte con el oso perezoso de dos dedos C. hoffmanni (Salgado, 2010). La vejiga del tamandúa tiene un grosor de pared similar al descrito en caninos (Halásc, 2004), siendo de menor grosor al de C. hoffmanni (Salgado, 2010).

El estómago se pudo visualizar caudal al hígado en el abdomen medio, similar a caninos y osos perezosos de dos dedos $(C$. hoffmanni), en tanto que el grosor de la pared estomacal del tamandúa es menor a la de C. hoffmanni (Salgado, 2010), pudiendo deberse al diferente tipo de alimentación, siendo el tamandúa un insectívoro y más parecido al canino (carnívoro) que al oso perezoso de dos dedos (herbívoro forrajero).

El hígado en el tamandúa, al igual que en el canino, está localizado principalmente en la región xifoidea y los hipocondrios derecho e izquierdo del abdomen (Scholz y
Lüerssen, 2000). A diferencia de otras especies de Xenarthras, como C. hoffmanni que presenta el hígado hipoecogénico con relación al riñón, el tamandúa presentó el hígado hiperecogénico en comparación al riñón, y la vesícula biliar en forma ovalada, similar al descrito en caninos (Mamprim, 2004).

$\mathrm{Al}$ igual que en caninos, el parénquima esplénico es hiperecogénico con relación a la corteza renal, presenta forma alargada, se ubica en el flanco izquierdo y presenta una cápsula fina lisa bien definida y ecogénica (Gomes, 2004). Los testículos, al igual que en otros Xenarthras como el C. hoffmanni, se encuentran intraabdominales, característica que se puede atribuir al ser animales de baja temperatura y que, por lo tanto, requieren aumentar la temperatura testicular en la cavidad abdominal para mantener la espermatogénesis.

El empleo de esta técnica imagenológica puede ser de utilidad para el sexaje en la especie, sugiriéndose su empleo, dado que la técnica tradicional basada en el dimorfismo sexual (Rossi et al., 2013) puede no resultar determinante para demostrar el sexo, debido al pequeño tamaño de los órganos genitales externos.

\section{Conclusiones}

- Las características ecográficas de los órganos abdominales de los cinco especímenes de Tamandua tetradactyla muestran que esta especie comparte características similares con caninos domésticos de similar talla y peso.

- La topografía de los riñones y testículos difieren al de caninos domésticos. Tiene los riñones ubicados entre las regiones del flanco e inguinal y los testículos intraabdominales, lo cual comparte con otras especies del superorden Xenarthra. 


\section{Literatura Citada}

1. Alves da Rosa C, Hobus Q, Bager A. 2010. Mammalia, Pilosa, Myrmecophagidae, Tamandua tetradactyla (Linnaeus, 1758): Distribution extension. Check List 6(1). [Internet]. Available in: https://www.biotaxa.org/cl/article/view/ 6.1.052. doi: $10.15560 / 6.1 .052$

2. Barr F. 2006. Ultrasound versus radiology of the abdomen. The North American Veterinary Conference 2006: 594-595. [Internet]. Available in: www.ivis.org/proceedings/navc/2006/ SAE/207.asp?LA=1

3. Bonatelli M, Papa P, Santos TC, Miglino MA, Passipieri M, Ambrósio CE, Carvalho AF, et al. 2003. Morfología dos orgâos genitais femeninos do tamandua bandeira, Myrmecophaga tridactyla. En: IV Congreso de Anatomía del Cono Sur. Brasil. doi: 10.4067/ S0717-95022003000100008

4. Diniz LS, Costa EO, Oliveira PM. 1995. Clinical disorders observed in anteaters (Myrmecophagidae, Edentata) in captivity. Vet Res Commun 19: 409-415. doi: 10.1007/BF01839320

5. Fernandes TN, Young RJ. 2008. Fluctuations in the tympanic membrane temperatures of non-restrained captive giant anteaters and southern tamanduas. J Zoology 274: 94-98.

6. Gardner A. 2007. Family Myrmecophagidae. En: Gardner A (ed). Mammals of South America: marsupials, xenarthrans, shrews and bats. Vol1. USA: University of Chicago Press. p 171-175.

7. Gomes V. 2004. Baço. En: Carvalho F (ed). Ultrasonografia en pequenos animais. Brasil: Roca. p 85-93.

8. Halásc M. 2004. Sistema urinário: rins, ureteres, bexiga urinária e uretra. En: Carvalho F (ed). Ultrasonografia en pequenos animais. Brasil: Roca. p 111-146.

9. Hayssen V. 2011. Tamandua tetradactyla (Pilosa: Myrmecophagidae). Mammalian Species 43: 64-74. doi: $10.1644 / 875.1$
10. Howard EE, De Lahunta A. 1991. Miller, disección del perro. Pennsylvania, EEUU: Interamericana Mac Graw-Hill. $369 \mathrm{p}$.

11. Janthur M, Lüerssen D. 2000. Kidneys and ureters. In: Poulsen C, Tobias R (eds). An atlas and textbook of diagnostic ultrasonography of the dog and cat. Veterinary Learning Systems. p. 210-227.

12. Macedo BC, De Lima AR, Pereira, Corrêa L, Giese EG, Mattos E, Branco E. 2011. Aspectos morfométricos e morfológicos macro e microscópicos dos intestinos do Tamandua tetradactyla, En: Anais do $9^{\circ}$ Seminário Anual de Iniciação Científica. Belém, Brasil.

13. Mamprim MJ. 2004. Figado e vesicula biliar. En: Carvalho C (ed). Ultrasonografia en pequenos Animais. Brasil: Roca. p 51-73.

14. Mannion $P$. 2006. Diagnostic ultrasound in small animal practice. Ed Blackwel. 338 p. doi: 10.1002/9780470752357

15. Miranda Luppi M, Teixeira da Costa ME, Cordeiro M, Cancado R. 2008. Deficiencia de taurina em filhote de Tamanduá-mirim (Tamandua tetradactyla) criado com substitutos de leite para cães e gatos. Ciênc Anim Brasileira 9: 1004-1009.

16. Moreno G. 2012. Use of dexmedetomidine, midazolam, ketamine and reversal with atipamezol for chemical immobilization on giant anteaters (Myrmecophaga tridactyla), lesser anteaters (Tamandua tetradactyla) and silky anteaters (Cyclopes didactylus) kept in captivity. In: 44 ${ }^{\text {th }}$ Annual Conference American Association of Zoo Veterinarians. Oakland, CA AZV, Oakland, CA.

17. Pérez G, González G. 2004. Evaluación de una dieta para tamanduás (Tamandua spp) utilizadas en el Jardín Zoológico de Rosario, Argentina y el Zoológico La Aurora, Guatemala. Edentata 6: 4350. doi: 10.1896/1413-4411.6.1.43 
18. Rossi LF, Rhon Calderón E, Alonso FM, Luaces JP, Merani MS. 2013. Observaciones anatómicas e histológicas del sistema reproductor masculino y femenino en Tamandua tetradactyla (Myrmecophagidae: Xenarthra). InVet 15: 17-28.

19. Salgado S. 2010. Estudio ecográfico de los órganos abdominales del oso perezoso de dos dedos (Choloepus hoffmanni). Tesis de Médico Veterinario Zootecnista. Lima, Perú: Univ. Peruana Cayetano Heredia. 34 p.

20. Sanches TC, Miranda FR, Oliveira AS, Matushima ER. 2013. Hematology values of captive giant anteaters (Myrmecophaga tridactyla) and collared anteaters (Tamandua tetradactyla). Pesq Vet Bras 33: 557-560. doi: 10.1590/ S0100-736X2013000400022

21. Scholz S, Lüerssen D. 2000. Liver and gallbladder. En: Poulsen C, Tobias R (eds). An atlas and textbook of diagnostic ultrasonography of the dog and cat. Veterinary Learning Systems. p 181-197.

22. Soto AT, Gómez V, Migliorisi L, Laplace R. 2006. Diagnóstico de gestación por ultrasonografía en tamanduá (Tamandua tetradactyla). En: VIII Jornadas de Divulgación Técnico-Científicas. Facultad de Ciencias Veterinarias, Universidad Nacional de Rosario. Argentina.

23. Superina M, Miranda FR, Abba A. 2010. The 2010. anteater red list assessment. Edentata 11: 96-114. doi: $10.5537 / 020.011 .0201$

24. Superina M, Miranda F, Plese T. 2008. Maintenance of xenarthra in captivity. En: Vizcaíno SF, Loughry WJ (eds). The biology of the Xenarthra. Gainesville, USA: University Press of Florida. p 232-243.

25. Valdes E, Brenes A. 2012. Feeding and nutrition of anteaters. In: Fowler's zoo and wild animal medicine. Current therapy. Elsevier Saunders. p 378-383.

26. Vogt P, Becker C. 1987. Zur ersten Aufzucht eines Tamanduas (Tamandua tetradactyla) im Kefelder Zoo. Zool Gart 57: 221-233.

27. Zanzonico P. 2011. Noninvasive imaging for supporting basic research. In: Kiessling F, Pichler B (eds). Small animal imaging. Berlin: Springer. p 3-16. 